\title{
INERTIAL ELASTIC INSTABILITY OF ROTATING NANO DISKS
}

\author{
UĞUR GüVEN \\ Gümüssuyu PTT, PK 18, Istanbul, Turkey \\ e-mail: uguven@yildiz.edu.tr
}

\begin{abstract}
In this work, the static inertial-elastic instability of rotating nano disks is investigated with the centrifugal force formulation considering the radial displacement. Thus, Brunelle's previous local solution is generalized by using Eringen's nonlocal elasticity theory. The variations of critical rotation speeds with the nonlocal scale parameter are illustrated under different boundary conditions. It is seen that the critical rotation speeds decrease as the nonlocal scale parameters increase for all cases. Also, it is remarkable that the presented results are affected significantly from the boundary conditions.
\end{abstract}

Keywords: nonlocal elasticity, inertial instability, rotating disk, critical speeds

\section{Introduction}

The existence of static inertial-elastic instability in rotating disks has been noticed firstly by Brunelle (1971). In his analysis, the centrifugal body force was taken to be including the radial displacement; $\rho \Omega^{2}(r+u)$ where $\rho$ is mass density, $\Omega$ is rotational speed, $r$ is initial undeformed radius and $u$ is radial displacement. He reported that this type of instability has primary importance in rotating structures made of low modulus and high-yield strength materials. Hence, in such cases, the centrifugal body force can be modified as Brunelle suggested, and this modified form of the centrifugal body force can be seen to be reasonable. By taking as the starting point Brunelle's suggestion in regard to the calculation of the centrifugal force, so far various interesting papers with different purposes for rotating disks and cylinders have been published. The effects of finite deformation upon a rotating orthotropic cylinder with linear elasticity were investigated (Sandman, 1974). A new approach was presented (Güven, 1992) for the analysis of transverse vibrations of rotating disks having equal strength. The effects of anisotropy on the inertio-elastic instability of rotating disks and cylinders were investigated (Tutuncu, 2000; Tutuncu and Ozturk, 2004) analytically. After a lot of years, Brunelle's analytical results were verified with ANSYS (Chianese, 2011). Recently, the inertio-elastic instability analysis of FGM rotating disks of variable thickness fixed to a rigid shaft has been addressed in detail by using complementary functions method (CFM) (Yildirim and Tutucu, 2018).

The recent developments in science and technology have enabled possibility of production and use of various rotating structures in micro and nano scales. Recently, the static resonance of rotating nanobars was investigated (Güven, 2018) based on Eringen's nonlocal elasticity theory. However, no analytical or numerical studies have been done up to date on the static inertio elastic instability problem of rotating nano disks.

The present analysis tackles the inertial-elastic instability problem of rotating thin nano disks. The analysis is based on Eringen's nonlocal elasticity theory and Brunelle's idea for calculation of the centrifugal bod force which includes the radial displacement. In the analysis, three different cases are investigated; a hollow rotating disk clamped at the inner edge and free at the outer edge, and a hollow rotating disk free at the inner and outer edges and a rotating solid disk free at the outer edge. The closed form analytical solutions and relationships between the critical rotation speeds and size dependent nonlocal scale parameter are presented. 


\section{Basic relations and closed form analytical solutions}

In the present analysis, a thin homogeneous and isotropic rotating nano disk is considered. Also, following Brunelle's approach in regard to the centrifugal body force, the equation of motion in terms of stresses can be expressed as

$$
\frac{d \sigma_{r r}}{d r}+\frac{\sigma_{r r}-\sigma_{\theta \theta}}{r}+\rho \Omega^{2}(r+u)=0
$$

where $\sigma_{r r}$ and $\sigma_{\theta \theta}$ are the radial and tangential stresses, respectively.

In this analysis framework, the strain components in terms of radial displacement $u$ are

$$
\varepsilon_{r r}=\frac{d u}{d r} \quad \varepsilon_{\theta \theta}=\frac{u}{r}
$$

A basis non-local constitutive equation was derived as (Eringen, 1983)

$$
\boldsymbol{\sigma}-\left(e_{o} a\right)^{2} \nabla^{2} \boldsymbol{\sigma}=\mathbf{C}: \boldsymbol{\varepsilon}
$$

where $e_{0} a, \boldsymbol{\sigma}, \boldsymbol{\varepsilon}$ and $\mathbf{C}$ denote the size dependent small scale coefficient, the stress tensor, the strain tensor and the fourth order elastic modulus tensor, respectively.

The all nonlocal constitutive formulations can be obtained from above Eq. (2.3). Thus, the stress-radial displacement relations for the axial symmetric case in the cylindrical coordinate considering Eqs. (2.2) and (2.3) can be derived as

$$
\begin{aligned}
\sigma_{r r}-\left(e_{o} a\right)^{2}\left(\nabla^{2} \boldsymbol{\sigma}\right)_{r r} & =\frac{E}{1-\nu^{2}}\left(\frac{d u}{d r}+\nu \frac{u}{r}\right) \\
\sigma_{\theta \theta}-\left(e_{o} a\right)^{2}\left(\nabla^{2} \boldsymbol{\sigma}\right)_{\theta \theta} & =\frac{E}{1-\nu^{2}}\left(\frac{u}{r}+\nu \frac{d u}{d r}\right)
\end{aligned}
$$

where $\nu$ is the Poisson ratio, $\left(\nabla^{2} \boldsymbol{\sigma}\right)_{r r}$ and $\left(\nabla^{2} \boldsymbol{\sigma}\right)_{\theta \theta}$ denote the radial and tangential components of Laplacian of the stress tensor, respectively, in the cylindrical coordinates. These components for the axial symmetric case are given as (Povstenko, 1995; Yu and Lim, 2013; Ghavanloo et al., 2014; Tufekci and Aya, 2016)

$$
\begin{aligned}
& \left(\nabla^{2} \sigma\right)_{r r}=\nabla^{2} \sigma_{r r}-\frac{2}{r^{2}}\left(\sigma_{r r}-\sigma_{\theta \theta}\right) \\
& \left(\nabla^{2} \sigma\right)_{\theta \theta}=\nabla^{2} \sigma_{\theta \theta}+\frac{2}{r^{2}}\left(\sigma_{r r}-\sigma_{\theta \theta}\right)
\end{aligned}
$$

where

$$
\nabla^{2} \sigma_{r r}=\frac{d^{2} \sigma_{r r}}{d r^{2}}+\frac{1}{r} \frac{\sigma_{r r}}{d r} \quad \nabla^{2} \sigma_{\theta \theta}=\frac{d^{2} \sigma_{\theta \theta}}{d r^{2}}+\frac{1}{r} \frac{\sigma_{\theta \theta}}{d r}
$$

In the meantime, it should be mentioned that in some papers (e.g. Kiani, 2012a,b), constitutive nonlocal Eqs. (2.4) have been wrongly expressed as follows

$$
\begin{aligned}
\sigma_{r r}-\left(e_{o} a\right)^{2} \nabla^{2} \sigma_{r r} & =\sigma_{r r}^{\text {local }} \\
\sigma_{\theta \theta}-\left(e_{o} a\right)^{2} \nabla^{2} \sigma_{\theta \theta} & =\sigma_{\theta \theta}^{\text {local }}
\end{aligned}
$$

Using Eqs. (2.4) and (2.5), the nonlocal differential equation of motion in terms of the stresses and radial displacement is obtained as

$$
\begin{aligned}
\frac{d \sigma_{r r}}{d r} & +\frac{\sigma_{r r}-\sigma_{\theta \theta}}{r}-\left(e_{o} a\right)^{2}\left[\nabla^{2}\left(\frac{d \sigma_{r r}}{d r}+\frac{\sigma_{r r}-\sigma_{\theta \theta}}{r}\right)-\frac{1}{r^{2}}\left(\frac{d \sigma_{r r}}{d r}+\frac{\sigma_{r r}-\sigma_{\theta \theta}}{r}\right)\right] \\
= & \frac{E}{1-\nu^{2}}\left(\frac{d^{2} u}{d r^{2}}+\frac{1}{r} \frac{d u}{d r}-\frac{u}{r^{2}}\right)
\end{aligned}
$$


Having substituted Eq. (2.1) into Eq. (2.8), nonlocal differential equation (2.8) becomes as follows

$$
r^{2} \frac{d^{2} u}{d r^{2}}+r \frac{d u}{d r}+\left[\bar{\Omega}_{1}^{2}\left(\frac{r}{b}\right)^{2}-1\right] u=-\bar{\Omega}_{1}^{2} \frac{r^{3}}{b^{2}}
$$

where $b$ is the outer radius and

$$
\bar{\Omega}_{1}^{2}=\frac{\bar{\Omega}^{2}}{1-\left(e_{0} a\right)^{2} \bar{\Omega}^{2}} \quad \bar{\Omega}^{2}=\frac{\left(1-\nu^{2}\right) \rho \Omega^{2} b^{2}}{E}
$$

Equation (2.9) is a familiar Bessel equation with the general solution (Watson,1966; McLachlan, 1961)

$$
\frac{u}{b}=A J_{1}\left(\bar{\Omega}_{1} \frac{r}{b}\right)+B Y_{1}\left(\bar{\Omega}_{1} \frac{r}{b}\right)-\frac{r}{b}
$$

where $A$ and $B$ are undetermined integration constants, $J_{1}$ and $Y_{1}$ are Bessel functions.

An alternative solution can be developed following a different way. For this, nonlocal constitutive Eqs. (2.4) should be expressed in modified forms as follows

$$
\begin{aligned}
& \sigma_{r r}-\left(e_{o} a\right)^{2} l^{2} \sigma_{r r}=\sigma_{r r}^{\text {local }}=\frac{E}{1-\nu^{2}}\left(\frac{d u}{d r}+\nu \frac{u}{r}\right) \\
& \sigma_{\theta \theta}-\left(e_{o} a\right)^{2} l^{2} \sigma_{\theta \theta}=\sigma_{\theta \theta}^{\text {local }}=\frac{E}{1-\nu^{2}}\left(\frac{u}{r}+\nu \frac{d u}{d r}\right)
\end{aligned}
$$

where $l^{2}$ is a differential operator and it is defined as $l^{2}=\left(d^{2} / d r^{2}\right)+(1 / r)(d / d r)-\left(1 / r^{2}\right)$. Substituting Eqs. (2.11) into Eq. (2.1), one can recover directly Eq. (2.9).

The integration constants $A$ and $B$ are determined from the boundary conditions for three different cases addressed in this analysis. Firstly, for the clamped-free hollow disk from the related boundary conditions $u(a)=\sigma_{r r}(b)=0$ by using Eqs. (2.10) and $(2.11)_{1}$, these constants are determined as

$$
\begin{aligned}
& A=\frac{1}{C_{c r}}\left\{(1+\nu) Y_{1}\left(\bar{\Omega}_{1} \frac{a}{b}\right)-\frac{a}{b}\left[\bar{\Omega}_{1} J_{0}\left(\bar{\Omega}_{1}\right)-(1-\nu) J_{1}\left(\bar{\Omega}_{1}\right)\right]\right\} \\
& B=\frac{1}{C_{c r}}\left\{\frac{a}{b}\left[\bar{\Omega}_{1} J_{0}\left(\bar{\Omega}_{1}\right)-(1-\nu) J_{1}\left(\bar{\Omega}_{1}\right)\right]-(1+\nu) J_{1}\left(\bar{\Omega}_{1} \frac{a}{b}\right)\right\}
\end{aligned}
$$

where

$$
C_{c r}=Y_{1}\left(\bar{\Omega}_{1} \frac{a}{b}\right)\left[\bar{\Omega}_{1} J_{0}\left(\bar{\Omega}_{1}\right)-(1-\nu) J_{1}\left(\bar{\Omega}_{1}\right)\right]-J_{1}\left(\bar{\Omega}_{1} \frac{a}{b}\right)\left[\bar{\Omega}_{1} Y_{0}\left(\bar{\Omega}_{1}\right)-(1-\nu) Y_{1}\left(\bar{\Omega}_{1}\right)\right]
$$

Secondly, for the free-free hollow disk, from the corresponding boundary conditions $\sigma_{r r}(a)=$ $\sigma_{r r}(b)=0$ and by using Eq. $(2.11)_{1}, A$ and $B$ are determined as

$$
\begin{aligned}
& A=(1+\nu) \frac{1}{C_{c r}}\left\{\left[\bar{\Omega}_{1} Y_{0}\left(\bar{\Omega}_{1}\right)-(1-\nu) Y_{1}\left(\bar{\Omega}_{1}\right)\right]-\left[\bar{\Omega}_{1} Y_{0}\left(\bar{\Omega}_{1} \frac{a}{b}\right)-\frac{b}{a}(1-\nu) Y_{1}\left(\bar{\Omega}_{1}\right) \frac{a}{b}\right]\right\} \\
& B=(1+\nu) \frac{1}{C_{c r}}\left\{\left[\bar{\Omega}_{1} J_{0}\left(\bar{\Omega}_{1} \frac{a}{b}\right)-\frac{b}{a}(1-\nu) J_{1}\left(\bar{\Omega}_{1} \frac{a}{b}\right)\right]-\left[\bar{\Omega}_{1} J_{0}\left(\bar{\Omega}_{1}\right)-(1-\nu) J_{1}\left(\bar{\Omega}_{1}\right)\right]\right\}
\end{aligned}
$$

and

$$
\begin{aligned}
C_{c r} & =\left[\bar{\Omega}_{1} J_{0}\left(\bar{\Omega}_{1} \frac{a}{b}\right)-\frac{b}{a}(1-\nu) J_{1}\left(\bar{\Omega}_{1} \frac{a}{b}\right)\right]\left[\bar{\Omega}_{1} Y_{0}\left(\bar{\Omega}_{1}\right)-(1-\nu) Y_{1}\left(\bar{\Omega}_{1}\right)\right] \\
& -\left[\bar{\Omega}_{1} J_{0}\left(\bar{\Omega}_{1}\right)-(1-\nu) J_{1}\left(\bar{\Omega}_{1}\right)\right]\left[\bar{\Omega}_{1} Y_{0}\left(\bar{\Omega}_{1} \frac{a}{b}\right)-\frac{b}{a}(1-\nu) Y_{1}\left(\bar{\Omega}_{1}\right) \frac{a}{b}\right]
\end{aligned}
$$

Thirdly, for the solid disk, the integration constant $B$ must be zero in order that the stresses are finite at the centre. The second boundary condition $\sigma_{r r}(b)=0$ gives

$$
A=\frac{1+\nu}{C_{c r}} \quad C_{c r}=\bar{\Omega}_{1} J_{0}\left(\bar{\Omega}_{1}\right)-(1-\nu) J_{1}\left(\bar{\Omega}_{1}\right)
$$




\section{Numerical results}

In this Section, the effects of the size dependent small scale coefficient on the critical rotational speeds that cause the inertial elastic instability are addressed for different boundary conditions, i.e. clamped-free (C-F), free-free (F-F) hollow disks and the solid disk. The variations of dimensionless critical rotational speeds with the dimensionless nonlocal small scale coefficients are shown in Figs. 1-3, for C-F, F-F and the solid disk. The dimensionless quantities in the numerical illustrations are defined to be: the dimensionless nonlocal small scale coefficient $\left(e_{0} a\right) / b$, the dimensionless rotational speed $\left(1-v^{2}\right) \rho \Omega^{2} b^{2} / E$. The material properties like Poisson's ratio $\nu$, Young's modulus $E$ and density $\rho$ are taken to be $0.3,70 \mathrm{GPa}$ and $2700 \mathrm{~kg} / \mathrm{m}^{3}$, respectively. The outer radius $b$ is $1 \mathrm{~nm}$, and the assessments should be done in nano scale. In generally,

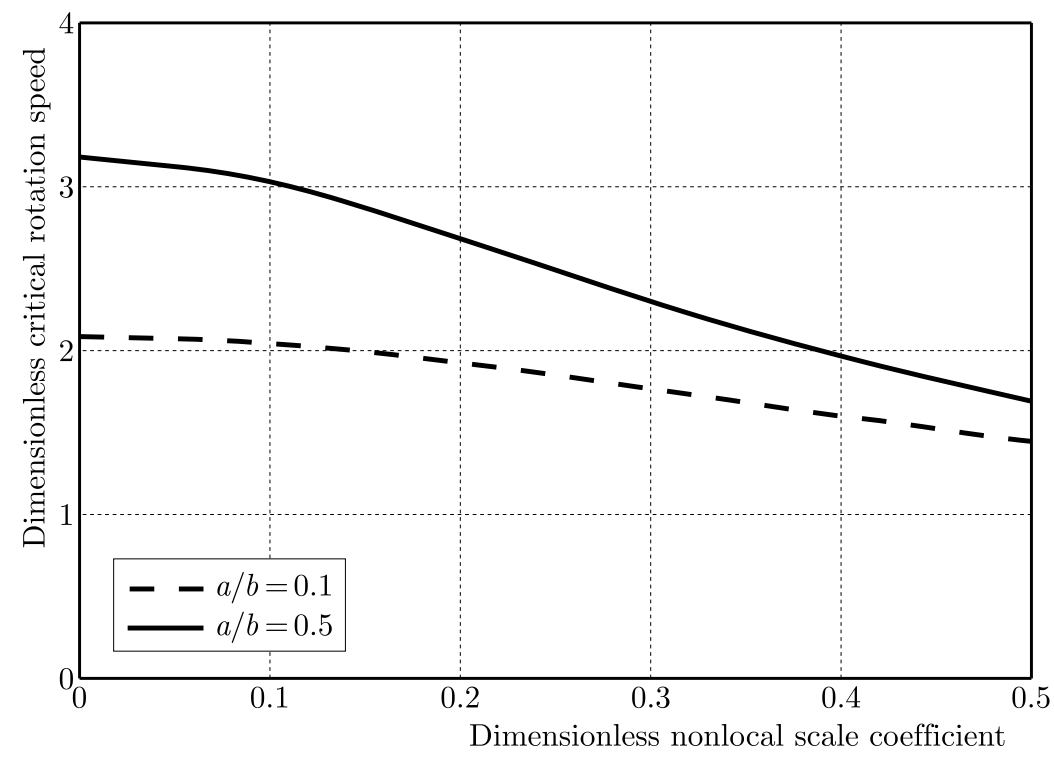

Fig. 1. Variation of the critical rotational speed with the nonlocal scale coefficient for the hollow disk under the clamped-free boundary condition

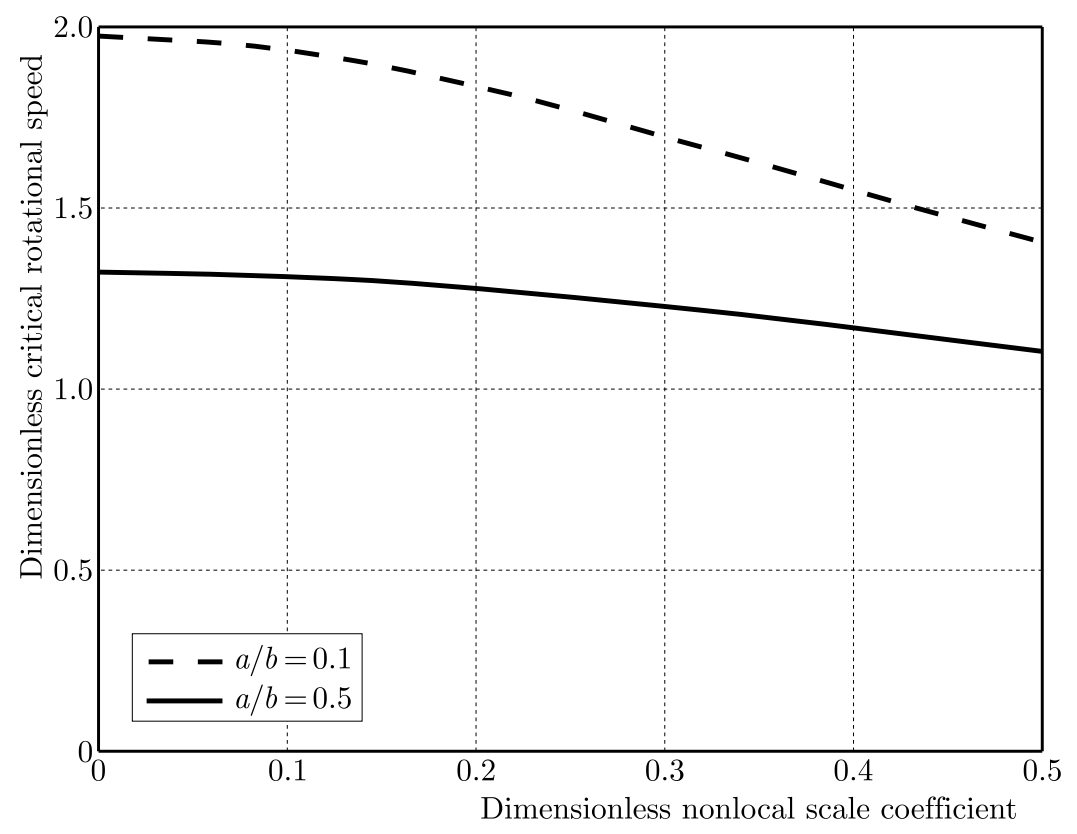

Fig. 2. Variation of the critical rotational speed with the nonlocal scale coefficient for the hollow disk under the free-free boundary condition 


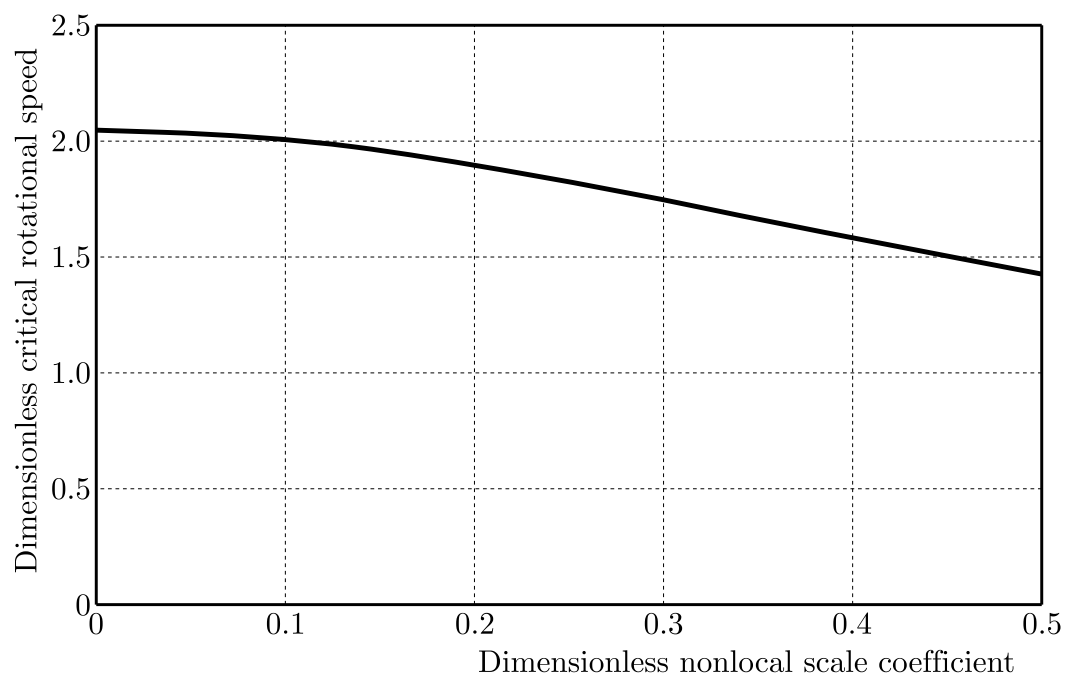

Fig. 3. Variation of the critical rotational speed with the nonlocal scale coefficient for the solid disk with the free edge

Figs. 1-3 show that with an increase in the size dependent small scale coefficients, the critical rotational speeds that lead to the inertial instability decrease. It is seen in a comparison between Figs. 1 and 2 that the critical speeds are significantly affected by the boundary conditions. This comparison shows that as the radii ratio increases, the critical rotation speeds increase, contrary to the free-free boundary conditions. These increases are more significant, especially in the low range of the scale coefficients. The critical rotation speeds under the clamped-free boundary conditions are more significantly affected by the small scale coefficients compared with the free-free boundary conditions. The inertial elastic instability occurs in smaller levels of the critical rotation speeds under free-free boundary conditions. The critical rotation speeds become insensitive versus the small scale coefficient as the radii ratio decreases under free-free boundary conditions.

\section{Conclusion}

In this work, the inertial elastic instability of rotating nano disks under different boundary conditions is investigated analytically. The analysis is based on the body force calculation including the radial displacement suggested by Brunelle. Using Eringen's nonlocal elasticity theory, the previous local solution is generalized for the nonlocal elasticity. In addition, this work includes other boundary conditions (i.e. free-free and solid disk) have not been reported in the previous literature. The nonlocal inertial stability analysis is discussed for different boundary conditions and radii ratios. The analysis provides useful insights for the rotating nano disks and shows that threat of the inertial instability becomes more critical as the radii decrease.

Dedicated to memory of Prof. Dr. Udo Gamer (1937-2017) from TU Wien, which was a wonderful man, an excellent researcher and academic teacher.

\section{References}

1. BRunelle E.J., 1971, Stress redistribution and instability of rotating beams and disks, America Institute Aeronautics Astronautics Journal, 9, 758-759

2. Chianese S., 2011, Safety factor against burst speed of turbomachinery rotating disks, M.Sc. Thesis, Department of Mechanical Engineering, University of Illinois, Chicago, U.S.A. 
3. ERIngen A.C., 1983, On differential equations of nonlocal elasticity and solutions of screw dislocation and surface waves, Journal of Applied Physics, 54, 4703-4708

4. Ghavanloo E., Fazelzadeh S.A., Rafin-Tabar H., 2014, Nonlocal Continuum-based modeling of breathing mode of nanowires including surface stress and surface inertia effects, Physica B, 440, 43-47

5. GüVEn U., 1992, On transverse vibrations of a rotating disk of uniform strength, Journal of Applied Mechanics, 59, 234-235

6. Güven U., 2018, Static resonance in rotating nanobars, Journal of Theoretical and Applied Mechanics, 56, 887-891

7. KiAni K., 2012a, Magneto-elasto-dynamic analysis of an elastically confined conducting nanowire due to an axial magnetic shock, Physics Letters A, 376, 1679-1685

8. Kiani K., 2012b, Magneto-thermo-elastic fields caused by an unsteady longitudinal magnetic field in a conducting nanowire accounting for eddy-current loss, Materials Chemistry and Physics, 136, $589-598$

9. McLachlan N.W., 1961, Bessel Functions for Engineers, Oxford, England: Clarendon Press

10. Povstenko Yu.Z., 1995, Circular dislocation loops in non-local elasticity, Journal of Physics D: Applied Physics, 28, 105-111

11. SAndman B.E., 1974, Finite deformation of a rotating orthotropic cylinder with linear elasticity, Computers and Structures, 4, 581-591

12. Tufekci E., AyA S.A., 2016. A nonlocal beam model for out-of-plane static analysis of circular nanobeams, Mechanics Research Communications, 76, 11-23

13. Tutuncu N., 2000, Effect of anisotropy on inertio-elastic instability of rotating disks, International Journal of Solids and Structures, 37, 7609-7616

14. Tutuncu N., Ozturk M., 2004, Stress redistribution and instability in orthotropic cylinders, Journal of Reinforced Plastics and Composites, 23, 941-950

15. Watson G.N., 1966, A Treatise on the Theory of Bessel Functions, Cambridge, England: Cambridge University Press

16. Yildirim S., Tutuncu N., 2018, On the inertio-elastic instability of variable-thickness functionally-graded disks, Mechanics Research Communications, 91, 1-6

17. Yu Y.M., Lim C.W., 2013, Nonlinear constitutive model for axisymmetric bending of annular graphene-like nanoplate with gradient elasticity enhancement effects, Journal of Engineering Mechanics, 139, 1025-1035 